\title{
RETOS Y POSIBILIDADES DE LOS CURSOS EN LÍNEAA PARTIR DE UNA EXPERIENCIA CONCRETA.
}

\section{CHALLENGES AND POSSIBILITIES OF ONLINE COURSES FROM ACTUAL EXPERIENCE.}

\author{
Ana Aurora Vidal Martínez \\ avidal@ciad.mx \\ Dra. Beatriz Olivia Camarena Gómez \\ betica@ciad.mx
}

Centro de Investigación en Alimentación y Desarrollo, A.C.

Carretera a la Victoria km. 0.6, Hermosillo Sonora (México)

Este trabajo retoma el modelo de McIsaac y Gunawardena (1996) para el diseño de interfaces de cursos en línea y revisa su eficacia en base a una experiencia. Se describe la plataforma utilizada y las competencias docentes deseables, así como el sistema tutorial brindado y la presentación/manejo de contenidos temáticos y materiales didácticos que dicha plataforma permite. Complementa este análisis los resultados de la experiencia en términos del grado de satisfacción de los participantes (estructura y diseño del curso) y el registro de actividad en la plataforma, información que se obtuvo mediante una encuesta de opinión en línea y la observación no participativa.

Palabras clave: Educación a distancia, medio de aprendizaje, sistema a tiempo real, tecnología educativa.

This paper recovers the model of McIsaac y Gunawardena (1996) for the interface design of online courses, and reviews their effectiveness based on an actual experience. The platform used is described: desirable teaching skills, tutorial system, presentation / thematic content management and materials. The analysis is completed with the results of the experience in terms of participants' satisfaction level (of the course structure and design) and the activity log on the platform, the information is obtained through an online opinion survey and nonparticipatory observation.

Keywords: Distance learning, learning environment, real time system, educational technology. 


\section{Introducción.}

En los últimos años, los avances presentados por las Tecnologías de la Información y la Comunicación (TIC) y las facilidades que éstas ofrecen para su aplicación práctica en diversos procesos de enseñanza-aprendizaje, han propiciado el incremento de la oferta de cursos de capacitación en línea, fenómeno social que afecta las relaciones humanas, delinea un amplio abanico de posibilidades a favor de la transmisión del conocimiento y demanda más competencias de tipo informático a docentes y estudiantes.

Si bien los cursos en línea siguen centrados en el usuario y en sus necesidades (Graham, 1993), en esta nueva cultura de la intercomunicación, las TIC, constituyen un novedoso puente de comunicación entre lo que se quiere enseñar y el estudiante. Al respecto, Adell y Castañeda (2010) afirman que las herramientas que ofrece Internet, combinadas con fórmulas tradicionales de comunicación, están transformando las estrategias de enseñanza-aprendizaje, al convertirse en la puerta de entrada y la estructura de apoyo en lo que respecta a la presentación, desarrollo e incluso evaluación de los cursos (Schwier \& Misanchuck, 1993). Arjona y Cebrián (2012), sugieren que al diseñar los cursos en línea se considere su utilidad en base al perfil de la «...población a la que se dirige, la formación general previa del alumnado y sus experiencias previas en cursos en línea» (p.104). Cacheiro (2011, p.79) señala que la formación del docente «...debe centrarse en la adquisición de competencias que permitan la integración de los recursos TIC». Mientras Castillo Arredondo y Cabrerizo Diago (2005), plantea reflexionar sobre el papel que juega el maestro en ese proceso, cómo se reorganiza e incluso adapta su papel de guía o asesor en la nueva cultura de la intercomunicación al trastocar el tradicional modelo enseñanza-aprendizaje por no ser tan necesaria su presencia física o corporal.

En tal contexto, este trabajo toma como referencia un curso en línea para explorar las ventajas, limitaciones y alternativas de la capacitación a distancia vía la descripción de los mecanismos y herramientas mediáticas que respaldan dicho curso y los resultados obtenidos con el mismo.

Primero, se describen algunos de los modelos educativos a distancia impulsados en México y España; después, el trabajo colegiado realizado por personal de distintas áreas para diseñar, desarrollar y difundir los cursos en línea. Posteriormente, se describen las competencias básicas de los docentes que participan en tales cursos; seguido de la descripción técnica básica que tales cursos requieren. Por último, la valoración de un curso en línea a partir de un instrumento que atiende tres variables: plataforma utilizada, proceso de enseñanza y sistema de tutorías que éste facilita.

\subsection{Tipo de modelos educativos a distancia y aspectos básicos de cursos en línea.}

Hoy en día, la educación en línea se encuentra en pleno proceso de crecimiento y evolución, es decir, hay buenos avances en las TIC y en los procesos técnicos que permiten su aplicación práctica, clara y sencilla, en diversos procesos de enseñanzaaprendizaje. Sin embargo, algunos autores señalan que esta aplicación tecnológica puede resultar contraproducente si los cursos carecen de un buen diseño y planificación 


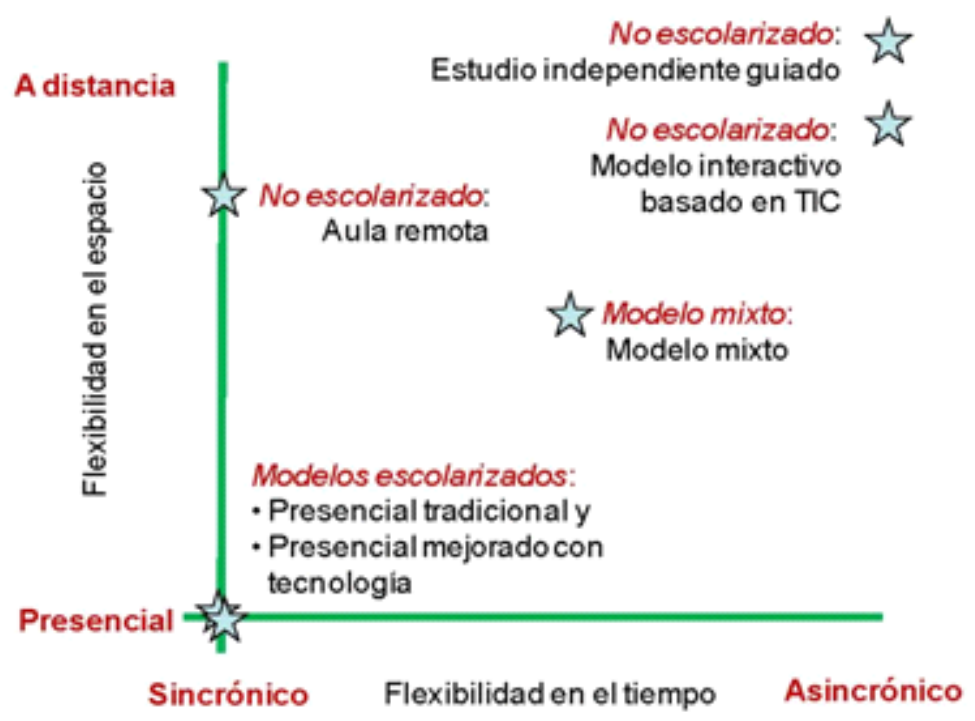

Figura 1. Grado de flexibilidad en el tiempo y espacio de los distintos modelos educativos, contrastándolos con el modelo presencial (CONACYT, 2012).

(McAnally-Salas, 2005). Hay que revisar entonces las características que a juicio de especialistas en educación y manejo de TIC debe poseer un curso en línea. Ahora bien, es posible distinguir varias modalidades de educación a distancia que tienen que ver con el tipo de interacción usuario-plataforma que cada una permite, tal y como se muestra a continuación.

En la Universidad Nacional de Educación a Distancia, la investigación titulada Cómo diseñar y ofertar cursos en línea (UNED, 2010), identificó tres niveles y tipos de interacción: 1) el nivel básico, cuya interacción usuarioplataforma es de tipo informativa, 2) el nivel intermedio, con interacción básica, y 3) el nivel avanzado, con interacción y actividades significativas entre usuario-plataforma. La razón por la cual es necesario hacer esta identificación de los niveles de integración de la virtualidad y los componentes tecnológicos en la oferta académica, es que ésta puede oscilar desde experiencias iniciales en las cuales la aproximación entre estudiantes y docentes es una mezcla entre lo presencial y la virtualidad, hasta las ofertas completas en línea. Tal nivel de integración de virtualidad y de componentes tecnológicos, dependerá del contexto y ubicación del curso en línea en el plan de estudios, de los contenidos, los objetivos de aprendizaje, del modelo evaluativo e incluso del perfil académico del estudiantes al que está dirigido el curso.

En México, al revisar los programas de posgrado a distancia que ofrece el Consejo Nacional de Ciencia y Tecnología (CONACYT, 2012) se distinguen al menos cinco tipos o modelos educativos: 1) el estudio independiente guiado (modalidad 
abierta); 2) el aula remota (modalidad a distancia); 3) el modelo interactivo basado en TIC (modalidad distancia); 4) el modelo híbrido (modalidad mixta); y 5) el modelo presencial apoyado con tecnología (modalidad presencial). Esta institución confirma que los factores que contribuyen en la calidad de un programa de posgrado a distancia o mixto tienen que ver con la preparación y desempeño de los docentes en tanto que han desarrollado las competencias para dicha modalidad educativa tanto en el diseño curricular del programa y en el diseño instruccional de las unidades de aprendizaje. En cada modelo, esos factores ocupan un lugar diferente en cuanto a la flexibilidad en el espacio y la flexibilidad en el tiempo (ver Figura 1).

También en la Universidad Autónoma de México (UAM), hay al menos cinco modelos en la gestión del conocimiento aplicados a la educación en línea, los cuales refieren a la relación entre estudiante y: a) texto didáctico, b) texto didáctico y docente, c) material didáctico, docentes y demás estudiantes, d) comunidad virtual de aprendizaje, e) docentes-estudiantes en eventos presenciales (Bañuelos \& Barrón, 2005).

De igual forma, la Universidad virtual del Tecnológico de Monterrey ha iniciado cursos en línea que guían al estudiante interesado para que sea capaz de avanzar hacia un aprendizaje auto-dirigido, significativo y colaborativo. Los usuarios de tales cursos cuentan con el apoyo del profesor, el tutor y se le ofrecen técnicas didácticas para reforzar su uso de TIC (Marcos, 2010). El estudiante es el centro de atención del proceso enseñanza-aprendizaje de los cursos virtuales y ese mismo modelo se usa como estrategia para fortalecer las competencias de los docentes.
En síntesis, los modelos adoptados por las instituciones educativas al incursionar en la modalidad a distancia al parecer han derivado de sus necesidades de articular las posibilidades que ofrecen las TIC con su particular contexto, especialidad y proceso educativo.

\subsection{Competencias deseables de los docentes.}

Para avanzar en el logro o efectividad de los cursos en línea, es deseable que los conductores del curso adquieran y muestren desarrollo en cinco competencias básicas (Salmon, 2004): acceso y motivación, socialización en línea, manejo e intercambio de información, construcción del conocimiento y desarrollo. A continuación, una breve descripción de ellas.

Para el acceso y motivación tanto los instructores (docentes) como los participantes (estudiantes) deben tener la posibilidad de acceder de manera ágil y sencilla al sistema en línea y estar motivados para hacerlo ya que para organizarse y participar habitualmente en cada actividad se requerirá de su compromiso, entusiasmo y esfuerzo. Lo que realmente importa aquí es que los participantes adquieran la capacidad emocional y social de aprender juntos en línea. Las recomendaciones son: inducir el uso de plataformas institucionales de aprendizaje en línea; comunicar los requerimientos del sistema para que las computadoras sean debidamente configuradas; orientar en el uso del nombre de usuario y contraseña de acceso al sistema; realizar actividades de motivación inicial (rompehielos, presentación de los participantes que incluyan al docente, entre otros); expresar las expectativas del curso; 
explorar la organización del curso en el entorno en línea; entre otras.

La competencia de socialización en línea trata de reforzar lazos de confianza y participación o involucramiento de los estudiantes en el curso. Se sugiere abrir espacios de comunicación social en los distintos módulos que componen el curso, que el tutor se involucre en las manifestaciones de los participantes, implementar normas que faciliten la comunicación, crear un ambiente agradable que sea propicio para la comunicación (de respeto, confianza y empatía).

La competencia manejo e intercambio de información pretende que el usuario aprenda a encontrar e intercambiar información de forma productiva y efectiva, incluso que sugiera nuevas actividades viables de realizar. Se recomienda desarrollar estrategias que generen independencia, entusiasmo y confianza alrededor del trabajo de investigación y búsqueda de información; actividades que potencien la interactividad con los contenidos del curso y su profundización, enlaces con la red, videoconferencias, biblioteca de medios, entre otros; establecer comunicación con todos los participantes (interacción), promover el intercambio de materiales y la colaboración.

El propósito de la construcción del conocimiento es que el usuario forme su propia interpretación de los conocimientos y sea capaz de enlazarlo con sus experiencias concretas. Lo deseable es que los tutores se transformen en autores en línea más que en sólo meros transmisores de información. Algunas acciones sugeridas para avanzar en ello son, entre otras, desarrollar actividades para que los estudiantes expongan sus ideas y resultados de investigación; crear espacios para el debate de ideas, solución colaborativa de problemas o proyectos y desarrollo o verificación de teorías en contextos o condiciones específicas; revisar los tópicos de discusión y la propuesta de nuevas líneas de conversación; el fin, motivar la crítica y el análisis.

En la competencia desarrollo se espera que los tutores dejen de cuestionarse cómo utilizar la participación en línea y la tomen con más dedicación y creatividad, es decir, que sean críticos y auto reflexivos. Para reforzarla, se sugiere proponer actividades que provoquen en los participantes el reconocimiento de los procesos y las experiencias que se llevan a cabo para construir significados; abrir espacios para la reflexión sobre cómo las redes de aprendizaje, el trabajo con otros y las oportunidades generadas en el curso, impactan en los procesos de aprendizaje; desarrollar actividades en las que se aplique lo aprendido, adaptándolas a diferentes contextos o situaciones; y reconocer los conflictos cognitivos y la resolución de ellos, a partir del cambio en las estructuras mentales como producto de una evolución y madurez adquirida durante el curso; entre otras.

Esta breve descripción de las competencias deseables que debe poseer el docente que participa en los cursos en línea, permite reconocer que éste sigue desempeñando un papel importante en el proceso enseñanzaaprendizaje. El uso de las TIC facilita la conexión a distancia entre los participantes en el proceso educativo sin desplazar el papel central, dinámico, que desempeñan docentes y estudiantes en cada actividad. La tipología del saber hacer reconoce el sistema de tutorías como una competencia docente, por tanto, tarea fundamental de cualquier plan de formación docente (Gallegos, Gámis \& Gutiérrez, 2010). En los cursos en línea, tal 
competencia incluye orientar al estudiante para que sea capaz de utilizar e incorporar adecuadamente las TIC en las actividades de enseñanza-aprendizaje.

\section{Método.}

Para valorar el diseño y estructura de la plataforma de los cursos en línea, el modelo de McIsaac y Gunawardena (1996), sugiere considerar seis características de las tecnologías: transmisión y acceso, control, interacción, características simbólicas del medio, la presencia social creada a través del medio, la interfaz entre el usuario y la máquina. Respecto al guía o facilitador de tales cursos, se señalan seis áreas formativas en ese ámbito de competencia: 1) desarrollo de habilidades en el uso de equipo tecnológico, 2) nuevas formas de organización de las tareas educativas, 3) capacidades para asumir un nuevo rol, 4) habilidades para planear adecuadamente dosificando el programa, 5) uso y elaboración de materiales y recursos didácticos electrónicos, así como, 6) conocimientos y habilidades para evaluar en ese nuevo modelo (González, 2010).

Sin embargo, como cada modelo educativo a distancia responde al contexto particular en el cual emerge (demanda, necesidades y situación concreta), habrá que ver cuáles de los recursos que ofrecen las TIC son idóneos y aplicables a las actividades educativas de interés, particularmente cuáles se retomarán y aplicarán en los cursos en línea. Habrá que revisar en qué situaciones la adopción de unas $u$ otras herramientas es pertinente y/o efectiva, es decir, garantizan el éxito del modelo enseñanza-aprendizaje. Este tipo de análisis implica revisar los llamados condicionantes básicos de tales cursos: la comunicación, la interacción y el involucramiento de interlocutores.

\subsection{Objetivo de la investigación.}

En este estudio se plantea conocer las ventajas, limitaciones y expectativas de la capacitación a distancia vía la descripción de los mecanismos y herramientas mediáticas que respaldan un curso en línea y la satisfacción de los estudiantes participantes. El análisis se realiza a través de tres variables: diseño de plataforma, pertinencia y contenido del curso, competencias docentes del guíainstructor del curso en línea. Interesa conocer si el sistema utilizado permite al estudiante familiarizarse con el manejo de la plataforma, desarrollar las actividades planteadas en el contenido del curso y explorar cómo el docente-guía-asesor refuerza esto último. Para el logro de los objetivos del curso es fundamental el manejo de sus contenidos, particularmente el desarrollo de las actividades y ejercicios planteados, los cuales se llevan a cabo a nivel individual pero también a través del dialogo e interacción. El docente, como figura física es corporalmente difusa pero su papel facilitador del proceso enseñanza-aprendizaje en este tipo de cursos es clave. En el guía o instructor recae la responsabilidad de reforzar la participación e interacción de y entre estudiantes, y con la plataforma, actúa como protagonista, mediador y facilitador de tal proceso.

\subsection{Procedimiento de la investigación.}

Se toma como referencia uno de los cursos en línea que ofreció el Centro de Investigación en Alimentación y Desarrollo, A.C. (CIAD, A.C.), en el verano del 2012, el cual fue desarrollado por el personal adscrito a 


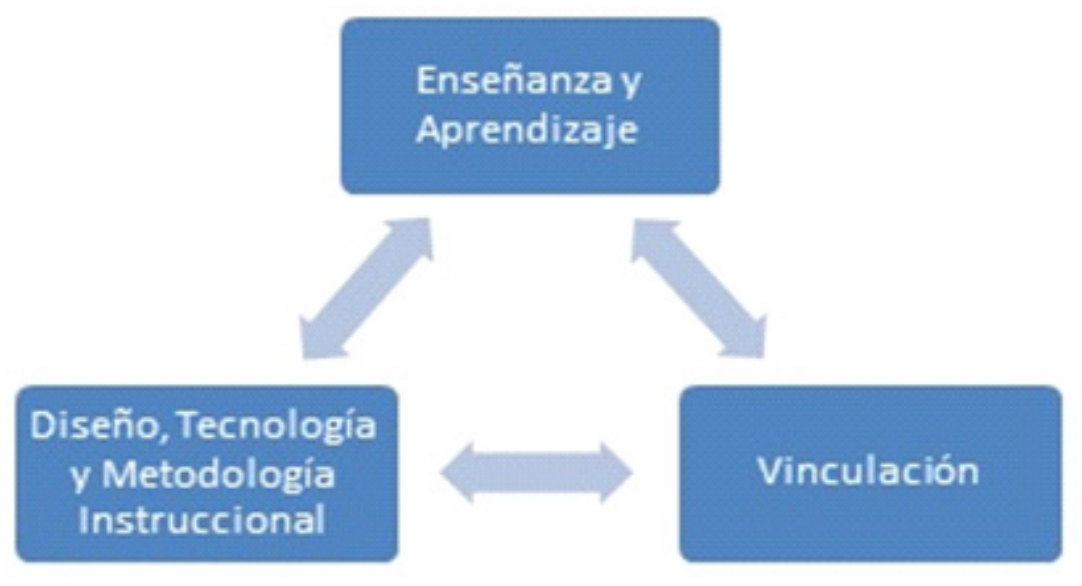

Figura 2. Áreas colaboradoras para llevar a cabo la impartición del curso en línea.

distintas áreas de trabajo de la institución. Generalmente participan tres áreas -es el caso del curso que se tomó como referencia-, la que define el contenido temático que sustenta el proceso enseñanza-aprendizaje, la que brinda el soporte tecnológicoinformático, y la responsable de la difusión del curso. Pueden sumarse otras, por ejemplo, la que sistematiza el desarrollo y resultado de cada curso, la que da seguimiento a los estudiantes, etc.

El área denominada Enseñanza y Aprendizaje, es la que define contenidos, procesos de evaluación y seguimiento del curso; pero también, cómo van a operar a distancia los procesos de instrucción, las tutorías y la atención de consultas. En el área Diseño, Tecnología y Metodología Instruccional, se cuida todo lo relativo a la selección de métodos y herramientas para el diseño del curso, material instruccional y soporte tecnológico que facilita el acompañamiento o asesoría del tutor/ docente (videos tutoriales, presentaciones dinámicas, etc.); se proporciona y pone en operación la infraestructura de equipos y sistemas que garantiza la comunicación interactiva entre instructores y alumnos y se provee el adiestramiento para el uso de tales herramientas tecnológicas. El área denominada Vinculación, establece los mecanismos para la venta y difusión de estos cursos.

En el caso analizado, esas atribuciones fueron tomadas por cada una de las tres áreas descritas. En la definición del contenido y estructura de los módulos de enseñanzaaprendizaje del curso en línea, participó personal de la coordinación, departamento o área que tiene facultades y conocimientos teórico-prácticos del tema de interés; quien participó como docente responsable del 
curso emergió de tal área. El apoyo o soporte para el uso de la tecnología y metodología instruccional de los cursos en línea, lo brindó el personal especializado adscrito al área de Informática o de TIC de la dependencia, por ser quienes poseen los conocimientos, habilidades y competencias para facilitar la inter-comunicación en línea de los participantes (estudiante-estudiante; estudiante-docente; docente-estudiantes). La institución también cuenta con un departamento o área de vinculación que fue la que apoyó en la difusión del curso. El trabajo fue colegiado, congruente con respecto al propósito y contenido del curso. Los involucrados participaron en distintos aspectos y momentos de la propuesta educativa, en su diseño, difusión, como tutor/ docente o soporte, con el apoyo logístico durante el desarrollo, monitoreo, seguimiento e incluso en la posterior evaluación del curso (ver Figura 2).

Los aspectos considerados para la implementación del curso en línea fueron: infraestructura tecnológica, requerimientos y compatibilidad de los equipos, nivel de interactividad necesario, personal de apoyo (tutores/conductores) y perfil de los estudiantes.

La tecnología utilizada fue una plataforma de código abierto denominada Moodle versión 1.9.4+. Se trata de un sistema de gestión de cursos de libre distribución (Course Management System CMS) que facilita crear comunidades de aprendizaje en línea. Moodle permitió articular el diseño haciendo uso de los respectivos módulos de foros, gestión de contenidos (recursos), cuestionarios con distintos tipos de preguntas, blogs, wikis, base de datos de actividades, encuestas, chat, glosarios, evaluación de pares, soporte de muchos idiomas (Sánchez, 2009).

Basado en reportes y recomendaciones de empresas independientes como BusinessSoftware (2012), WebConferencing (2011) y del análisis realizado por Urbina et al. (2010) se decidió utilizar Cisco Webex (2012), herramienta de colaboración a través de Internet que permite realizar video conferencias, enviar y recibir mensajería instantánea, y compartir archivos que pueden modificarse en línea e interactuar con ellos. El tipo de videoconferencia que ofrece Webex es conocido como de escritorio y a diferencia de lo que es una videoconferencia utilizando Messenger o Netmeeting, la de Webex permite interactuar con hasta veinticinco equipos a la vez y como la calidad de la imagen al compartir archivos es muy buena, facilita más la transferencia de conocimiento en línea.

Se diseñó una guía de estudios web para orientar al usuario sobre el contenido, objetivo y estructura del curso así como las actividades a realizar, la forma en la cual se establecerá la inter-comunicación y los requisitos técnicos mínimos a cumplir para evitar dificultades durante el desarrollo del curso. La información brindada tiene el fin de capacitar y entrenar a los participantes en el manejo de las dos herramientas tecnológicas que utilizarán durante el desarrollo del curso. Además del maestro especialista, participa un experto en el manejo de las herramientas informáticas para brindar soporte técnico a quien lo requiera, estudiantes o docentes.

Respecto al contenido del curso, éste se organizó en seis módulos, cada uno fue presentado semana a semana. La programación y duración efectiva del curso fue de seis semanas. Al cierre de cada módulo, se integró un procedimiento de autoevaluación y previamente fueron 


\begin{tabular}{|l|c|c|}
\hline \multirow{2}{*}{ VARIABLE } & OPCIÓN DE RESPUESTA & $\%$ \\
\hline \multirow{3}{*}{ Sexo } & Femenino & 52.2 \\
\cline { 2 - 3 } Escolaridad & Masculino & 47.8 \\
\cline { 2 - 3 } & Licenciatura & 52.2 \\
\cline { 2 - 3 } & Maestría & 13.0 \\
\hline \multirow{3}{*}{\begin{tabular}{l} 
Ocupación \\
\multirow{2}{*}{$\begin{array}{l}\text { Localidad de conexión } \\
\text { (entidad de México } \\
\text { ubicada en el ...) }\end{array}$}
\end{tabular}} & Doctorado & 8.7 \\
\cline { 2 - 3 } & Empleado & 17.4 \\
\cline { 2 - 3 } & Estudiante & 69.6 \\
\hline
\end{tabular}

Tabla 1. Perfil de Usuario/Participante en curso en línea.

programadas sesiones de videoconferencia de escritorio para la socialización de dudas de parte de los estudiantes al instructor y establecer diálogo entre ellos en el desarrollo de actividades diversas que reforzaran lograr el propósito del curso. El ingreso al sistema fue a través de una contraseña personal (previamente asignada) y la interacción con la plataforma fue posible las 24 horas del día.

Para explorar la experiencia y opinión de los participantes sobre el curso tomado, se utilizaron herramientas que remiten a la observación no participativa. Se trata de una observación con propósitos definidos, el investigador se vale de ella para obtener información y datos sin participar en los acontecimientos de la vida del grupo que estudia, es decir, permanece ajeno al mismo (Ander-Egg, 1983). Se programó la plataforma para llevar un registro de interacción por alumno y estar así en posibilidad de hacer una revisión sistemática de la intercomunicación lograda. Durante las seis semanas se recopilaron los reportes de las actividades en línea realizadas por estudiante, lo cual facilita describir la interacción que permite la plataforma diseñada.

La otra herramienta utilizada para valorar este curso en línea consistió en una encuesta de opinión en línea a través de un cuestionario que fue retomado y adaptado de la COLLES (Encuesta sobre ambiente de aprendizaje constructivista en línea) y del $A T T L S$ (Encuesta de actitudes de pensamiento y aprendizaje). Ambos instrumentos han mostrado ser útiles para evaluar y estimular el aprendizaje en contextos de aprendizaje en línea (Sánchez, 2009). El cuestionario consta de 21 reactivos agrupados en tres variables: enseñanza, materiales y sistema de tutorías. Hay preguntas abiertas, cerradas y tipo Likert. En éstas últimas, la opción de

Píxel-Bit. Revista de Medios y Educación. $N^{o}$ 44. Enero 2014. ISSN: 1133-8482. E-ISSN: 2171-7966. doi: http://dx.doi.org/10.12795/pixelbit.2014.i44.02 
respuesta varía de 1 a 5 , donde 1 es total desacuerdo, 2 desacuerdo, 3 ni en desacuerdo ni de acuerdo, 4 acuerdo y 5 total acuerdo. Se procede al análisis descriptivo y de tipo bivariante de la información obtenida destacando diferencias por sexo, escolaridad, ocupación y lugar geográfico de conexión al curso.

\subsection{Población que realizó el curso en línea.}

En el curso participaron 33 personas. Una para el diseño; una para la gestión administrativa; el maestro-experto en el tema; un asistente-guía del curso; tres para la difusión; y 23 usuarios-estudiantes.

Del perfil de los participantes fue posible identificar su sexo, escolaridad, ocupación, área disciplinaria de formación y región geográfica de conexión al curso. Por sexo, el $52 \%$ son mujeres y el resto hombres (48\%). En escolaridad, el 52\% cuenta con estudios de licenciatura, el $13 \%$ con estudios de maestría, el $8 \%$ con estudios de doctorado el 26\% de los usuarios no precisó esta información-. En ocupación, la mayoría están trabajando $(83 \%)$ y el resto son estudiantes (27\%) (ver Tabla 1).

En área disciplinaria de formación, anotaron Ingeniería química o Bioquímica, Biología y Química en alimentos. En localidad geográfica de conexión, mexicana en todos los casos, el $70 \%$ lo hizo de estados del norte del país (Sonora, Baja California y Sinaloa), el 26\% de entidades del centro (Nayarit, México, Jalisco y Aguascalientes), y el $4.3 \%$ restante del sur del país (Chiapas).

A continuación, lo más destacado de las respuestas vertidas en las variablesde investigación por cada grupo de interés.

\subsection{Análisis estadístico y discusión de resultados.}

Para la variable, diseño de plataforma: presentación, contenido, interactividad y material didáctico, la opinión promedio de los usuarios en esta variable fue 3.8, es decir, tienden a acordar que el diseño de la plataforma es adecuado, amigable, particularmente la presentación de contenidos temáticos y materiales didácticos, permite la interacción, participación y tutoría. Sin embargo, hay algunas diferencias interesantes al observar las respuestas vertidas por cada segmento de interés. Por sexo, la valoración de la plataforma por parte de los hombres supera ligeramente a la de las mujeres, ellos marcan acuerdo en casi todos los casos y ellas sólo tendencia a tal valoración (ver Tabla 2).

Por ocupación, son los estudiantes los que marcan acuerdo, -quizá por su familiaridad con este tipo de cursos y tecnologías-; y los empleados apenas tienden a tal valoración.

Por grado de escolaridad, se aprecia que a mayor nivel de estudios, mayor valoración de las bondades de la plataforma soporte del curso. En casi todos los casos la respuesta media de los usuarios con grado de doctor marca total acuerdo, la de quienes tienen grado de maestría marca acuerdo y la de quienes tienen grado de licenciatura tienden a ese valor e incluso en Utilicé la herramienta de comunicación para consultar al tutor sin ningún problema marcan indefinición.

La opinión de los grupos identificados por área geográfica, va de acuerdo a total acuerdo excepto en el ítem Utilicé la herramienta de comunicación para consultar al tutor sin ningún problema donde los del norte y del sur marcaron indefinición. Habrá que ver el porqué de tal opinión. 


\begin{tabular}{|c|c|c|c|c|c|c|c|c|c|c|c|}
\hline \multirow{3}{*}{ REACTIVO/CRITERIO } & \multicolumn{11}{|c|}{ RESPUESTA MEDIA DE POBLACIÓN (*) } \\
\hline & \multirow{2}{*}{ Total } & \multicolumn{2}{|c|}{ Por Sexo } & \multicolumn{2}{|c|}{ Por Ocupación } & \multicolumn{3}{|c|}{ Por Grado Escolar } & \multicolumn{3}{|c|}{ Por Entidad } \\
\hline & & $H$. & $M$ & Empl. & Estud. & Licencia. & Maest. & Doctora. & Nort & Centr & Sur \\
\hline $\begin{array}{l}\text { 11. La plataforma utilizada } \\
\text { en este curso fue amigable, } \\
\text { la pude utilizar sin } \\
\text { dificultad }\end{array}$ & 4.0 & 42 & 3.9 & 4.0 & 4.4 & 3.9 & 4.2 & 5.0 & 3.7 & 4.9 & 4.0 \\
\hline $\begin{array}{l}\text { 9. La distribución de los } \\
\text { materiales en el entorno } \\
\text { fue adecuada a mis } \\
\text { necesidades }\end{array}$ & 3.9 & 4.1 & 3.8 & 3.8 & 4.4 & 3.7 & 4.2 & 4.5 & 3.4 & 4.9 & 4.0 \\
\hline $\begin{array}{l}\text { 10. Los materiales que } \\
\text { ofrece la plataforma son } \\
\text { suficientes para facilitar la } \\
\text { comprensión de los temas }\end{array}$ & 3.8 & 4.2 & 3.9 & 3.7 & 4.4 & 3.6 & 4.2 & 5.0 & 3.6 & 4.4 & 4.0 \\
\hline $\begin{array}{l}\text { 12. La presentación de los } \\
\text { contenidos del curso fue } \\
\text { didáctica (facilita la } \\
\text { comprensión, guía los } \\
\text { aprendizajes, ejercita } \\
\text { habilidades y motiva) }\end{array}$ & 3.9 & 4.1 & 3.8 & 3.8 & 4.1 & 3.7 & 4.2 & 5.0 & 3.6 & 4.6 & 5.0 \\
\hline $\begin{array}{l}\text { 14. Mi participación e } \\
\text { intervención en el curso en } \\
\text { línea fue posible en todo } \\
\text { momento }\end{array}$ & 4.0 & 4.1 & 3.8 & 3.9 & 4.4 & 3.8 & 4.2 & 5.0 & 3.6 & 4.7 & 4.0 \\
\hline $\begin{array}{l}\text { 13. Utilicé la herramienta } \\
\text { de comunicación para } \\
\text { consultar al tutor sin } \\
\text { ningún problema }\end{array}$ & 3.4 & 3.7 & 3.4 & 3.3 & 3.9 & 3.2 & 3.8 & 4.5 & 3.2 & 4.0 & 3.0 \\
\hline
\end{tabular}

Tabla 2.Variable Diseño de Plataforma: contenido, interactividad, diseño, recursos didácticos.

(*) La respuesta varía de 1 a 5, donde 1=total desacuerdo; 2=desacuerdo; 3=ni de acuerdo ni en desacuerdo; 4= acuerdo; 5= total acuerdo.

\begin{tabular}{|c|c|c|c|c|c|c|c|c|c|c|c|}
\hline \multirow{3}{*}{ REACTIVO/CRITERIO } & \multicolumn{11}{|c|}{ RESPUESTA MEDIA DE POBLACIÓN (*) } \\
\hline & \multirow{2}{*}{ Total } & \multicolumn{2}{|c|}{ Por Sexo } & \multicolumn{2}{|c|}{ Por Ocupación } & \multicolumn{3}{|c|}{ Por Grado Escolar } & \multicolumn{3}{|c|}{ Por Entidad } \\
\hline & & $H$ & $M$ & Empl. & Estud. & Licencia. & Maest. & Doctora. & Nort & Centr & Sur \\
\hline $\begin{array}{l}\text { 1. Los conocimientos } \\
\text { adquiridos mediante la } \\
\text { capacitación a distancia } \\
\text { fueron excelentes }\end{array}$ & 3.8 & 4.0 & 3.6 & 3.8 & 3.6 & 3.7 & 3.5 & 5.0 & 3.6 & 4.3 & 4.0 \\
\hline $\begin{array}{l}\text { 5. La información } \\
\text { presentada en el curso } \\
\text { fue novedosa }\end{array}$ & 3.9 & 4.1 & 3.8 & 3.8 & 4.4 & 3.8 & 4.2 & 4.5 & 3.6 & 4.6 & 5.0 \\
\hline $\begin{array}{l}\text { 6. La planificación del } \\
\text { tiempo para llevar a cabo } \\
\text { el curso fue adecuada }\end{array}$ & 4.0 & 4.0 & 3.6 & 3.9 & 4.4 & 4.0 & 4.2 & 5.0 & 3.5 & 5.0 & 5.0 \\
\hline $\begin{array}{l}\text { 2. El contenido y } \\
\text { estructura de } \\
\text { presentación del curso } \\
\text { responde a las } \\
\text { necesidades de } \\
\text { conocimiento que plantea } \\
\text { mi práctica laboral } \\
\end{array}$ & 4.1 & 4.3 & 4.0 & 4.1 & 4.4 & 4.0 & 4.2 & 5.0 & 3.8 & 5.0 & 5.0 \\
\hline $\begin{array}{l}\text { 7. Las evaluaciones } \\
\text { realizadas fueron acordes } \\
\text { a los criterios } \\
\text { establecidos }\end{array}$ & 3.3 & 3.8 & 3.5 & 3.1 & 4.4 & 3.5 & 4.2 & 3.0 & 3.1 & 3.9 & 5.0 \\
\hline
\end{tabular}

Tabla 3. Variable Valoración del Curso: pertinencia, contenido y evaluación. 
En la variable valoración de pertinencia y contenido del curso la puntuación promedio es de 3.8, es decir, tendencia al acuerdo respecto a que el curso es pertinente, adecuado, novedoso, bien planificado, entre otros. Esta valoración cambia al observar las respuestas vertidas por sexo, escolaridad, ocupación y lugar de conexión (ver Tabla 3 ).

(*) La respuesta varía de 1 a 5, donde 1=total desacuerdo; $2=$ desacuerdo; $3=$ ni de acuerdo ni en desacuerdo; 4= acuerdo; 5= total acuerdo.

Por sexo, los hombres valoran más que las mujeres las bondades del curso en línea, los hombres marcan acuerdo en casi todos los reactivos y las mujeres tendencia.

Por ocupación, los estudiantes valoran mejor el curso que los empleados.

Por escolaridad, se aprecia de nuevo que a mayor nivel de estudios, más positiva es la valoración del curso en línea. Pero destaca también la indefinición en el ítem Utilicé la herramienta de comunicación para consultar al tutor sin ningún problemaen el grupo de estudiantes de licenciatura y de doctorado. Habrá que revisar con más detalle este punto.

Por entidad geográfica de conexión, la valoración es positiva en casi todos los ítems, más acentuada de parte de los usuarios del sur y del centro que los del norte. En estos últimos la respuesta media tiende al acuerdo e incluso en Utilicé la herramienta de comunicación para consultar al tutor sin ningún problema marcaron indefinición.

Para la variable valoración de instructor, sistema de tutoría e interactividad la puntuación promedio fue 3.8, es decir, tendencia al acuerdo con respecto a las aseveraciones aquí plasmadas del tutor, la interactividad y el sistema de tutoría que ofrece el curso (ver Tabla 4).
Al analizar las respuestas vertidas en esta variable por grupo de interés, se aprecia lo ya visto en las variables anteriores. Por sexo, los hombres lo valoran más positivamente que las mujeres. Por ocupación, los estudiantes más que los empleados. Por grado escolar, los que tienen estudios de doctorado, seguidos por los de maestría y finalmente los usuarios con estudios de licenciatura. Por entidad geográfica de conexión, los usuarios del centro del país, después los usuarios de los estados del sur y al último los usuarios del norte de México. En estos últimos predomina la indefinición al valorar al instructor (intervenciones bien estructuradas, asesoría adecuada, mantener la atención).

Para la variable relación de horas de actividad en la plataforma y calificación obtenida en el curso el sistema diseñado permite observar también, caso por caso, la relación existente entre horas actividad dedicadas al sistema y la calificación final obtenida. Tales registros permiten apreciar una relación directa entre horas de actividad y calificación obtenida: a más horas de actividad en línea la calificación es más alta. Si bien en la Gráfica 1, hay dos casos que saltan a la norma, al revisar su particular registro de actividad se aprecia que el participante con 25.26 horas de trabajo que obtuvo 0 de calificación, se mantuvo conectado a la plataforma moodle sin realizar actividad alguna; y el participante que presenta 18.4 horas de trabajo y obtuvo 98.5 de calificación, es investigador con bastante experiencia profesional en el tema del curso.

\section{Conclusiones.}

Las TIC han llegado para quedarse. Las instituciones académicas y de investigación 


\begin{tabular}{|c|c|c|c|c|c|c|c|c|c|c|c|}
\hline \multirow{3}{*}{ REACTIVO/CRITERIO } & \multicolumn{11}{|c|}{ RESPUESTA MEDIA DE POBLACIÓN (*) } \\
\hline & \multirow{2}{*}{ Total } & \multicolumn{2}{|c|}{ Por Sexo } & \multicolumn{2}{|c|}{ Por Ocupación } & \multicolumn{3}{|c|}{ Por Grado Escolar } & \multicolumn{3}{|c|}{ Por Entidad } \\
\hline & & $H$ & $M$ & Empl. & Estud. & Licencia. & Maest. & Doctora. & Nort & Centr & Sur \\
\hline $\begin{array}{l}\text { 4. El instructor demuestra } \\
\text { estar actualizado en la } \\
\text { materia }\end{array}$ & 4.0 & 4.1 & 3.8 & 4.0 & 4.4 & 4.0 & 4.2 & 5.0 & 3.6 & 5.0 & 5.0 \\
\hline $\begin{array}{l}\text { 17. En lo particular, } \\
\text { considero que las } \\
\text { respuestas brindadas por el } \\
\text { tutor durante el curso } \\
\text { fueron correctas y } \\
\text { adecuadas }\end{array}$ & 3.9 & 4.1 & 3.8 & 3.8 & 4.4 & 3.8 & 4.2 & 5.0 & 3.6 & 4.6 & 4.0 \\
\hline $\begin{array}{l}\text { 16. El instructor mantuvo } \\
\text { la atención de los } \\
\text { estudiantes durante las } \\
\text { sesiones }\end{array}$ & 3.7 & 4.0 & 3.7 & 3.6 & 4.4 & 3.5 & 4.2 & 4.5 & 3.4 & 4.3 & 4.0 \\
\hline $\begin{array}{l}\text { 15. Durante las sesiones de } \\
\text { videoconferencia el } \\
\text { instructor motivó la } \\
\text { participación de los } \\
\text { estudiantes }\end{array}$ & 4.0 & 4.1 & 3.8 & 3.9 & 4.1 & 3.9 & 3.8 & 4.5 & 3.5 & 5.0 & 5.0 \\
\hline $\begin{array}{l}\text { 8. El apoyo o asesoría } \\
\text { brindada en línea por el } \\
\text { tutor fue adecuada }\end{array}$ & 3.8 & 4.0 & 3.6 & 3.8 & 4.1 & 3.8 & 3.8 & 4.5 & 3.4 & 4.9 & 5.0 \\
\hline $\begin{array}{l}\text { 3. Las intervenciones del } \\
\text { instructor estuvieron bien } \\
\text { estructuradas }\end{array}$ & 3.7 & 3.9 & 3.5 & 3.7 & 3.9 & 3.6 & 3.5 & 4.5 & 3.4 & 4.4 & 3.0 \\
\hline
\end{tabular}

Tabla 4. Variable Valoración de Instructor, Sistema de Tutorías e Interactividad.

(*) La respuesta varía de 1 a 5, donde 1=total desacuerdo; 2=desacuerdo; 3=ni de acuerdo ni en desacuerdo; 4= acuerdo; 5= total acuerdo.

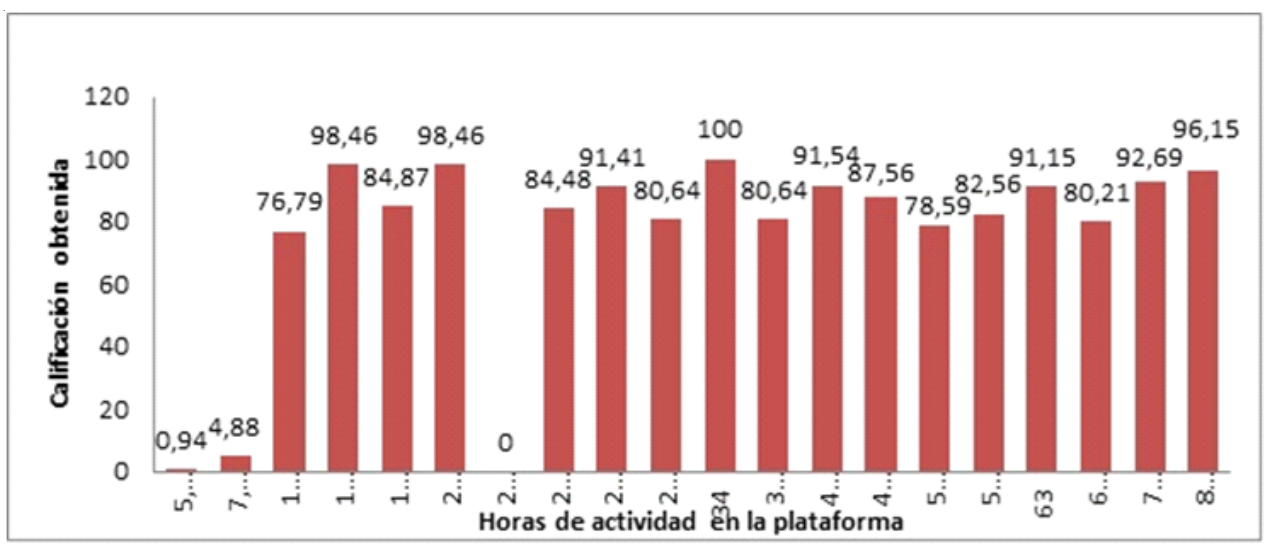

Grafica 1. Curso en Línea. Horas de actividad y calificación obtenida por participante. 
tendrán que aprovechar el nicho de oportunidad que las TIC abren en el terreno educativo; el riesgo de no hacerlo es quedarse rezagada en el mercado de la oferta y demanda educativa. Algunas ya han ingresado con cursos de especialización, talleres y hasta diplomados; pero persisten resistencias de parte del personal que está poco familiarizado con las TIC. Ese limitado manejo de las TIC por parte del personal y el poco avance de la organización educativa en tal proceso, no las exime de empezar a aprovechar las ventajas que la tecnología ofrece. Es recomendable que las instituciones promuevan cursos en línea en sus principales campos de especialidad. El apoyo institucional para avanzar en ese sentido es fundamental. Deben identificar al personal más competente (profesores/ investigadores) en el uso y manejo de TIC e invertir en la compra de programas y equipos que permita organizar los cursos desde cualquier sitio que disponga de Internet. Con la red celular digital multimedia se tiene la posibilidad de trasladarse de un sitio a otro. Con la tecnología 3G y 4G (tecnología de tercera y cuarta generación) se tiene acceso a un servicio de comunicaciones inalámbricas que permite estar conectado permanentemente a Internet garantizando la atención de zonas más distantes geográficamente.

En la experiencia del curso en línea descrita en este documento, se aprecia la valoración hecha por los usuarios. Se mostró que el modelo de McIsaac y Gunawardena (1996) ajustado al contexto y área temática de interés, resultó eficaz al menos en lo que respecta a la selección de las tecnologías a utilizar en el diseño de interfaces para cursos en línea, ya que la interactividad entre estudiantes y tutores se basó en el uso de tecnologías de Internet para el acceso a los materiales y para la interacción sincrónica y/ o asincrónica. En general, los usuarios del curso en línea valoraron positivamente las tres variables consideradas: diseño de plataforma (combinación de las tecnologías); contenido y pertinencia del curso; sistema de tutorías y desempeño del guía o tutor. Todos indicaron que previamente habían experimentado esta modalidad educativa. Sin embargo, hay algunas matizaciones en las respuestas vertidas por sexo, grado escolar, ocupación y lugar de conexión, que da pauta para la realización de estudios futuros que exploren esos aspectos de tipo contextual. En particular, destaca cierto perfil de quienes valoran mejor el curso: los hombres más que las mujeres; los estudiantes más que los empleados; los usuarios con grado de doctor o maestría; y los usuarios conectados desde el centro y sur del país. Se concluye que el curso en línea fue valorado positivamente por usuarios.

\section{Referencias bibliográficas.}

Adell Segura, J. \& Castañeda Quintero, L. (2010). Los Entornos Personales de Aprendizaje (PLEs): una nueva manera de entender el aprendizaje. En Roig Vila R. \& Fiorucci, M. (Eds.) Claves para la investigación en innovación y calidad educativas. La integración de las Tecnologías de la Información y la Comunicación y la Interculturalidad en las aulas. Stumenti di ricerca per I'innovaziones e la qualita ir ámbito educativo. La Tecnologie dell'informazione e della Comunicaziones e I'interculturalita nellas cuola. Alcoy: Marfil - Roma TRE Universita degli studi. 
Ander-Egg, E. (1983). Técnicas de investigación social: La observación. Buenos Aires: Humanitas.

Arjona Muñoz, J.A. \& Cebrián de la Serna, M. (2012). Expectativas y satisfacción de usuarios en cursos on line. Estudio del caso: experto en entornos virtuales de formación. Revista Píxel-Bit. Revista de Medios y Educación, 41, 93-107. Recuperado de http:/ /acdc.sav.us.es/pixelbit/images/stories/p41/ 07.pdf.

Bañuelos Márquez, A.M. \& Barrón Soto, H. (2005). Modelos de gestión del conocimiento para la educación en línea. Apertura, 5(1), 44-53.

Business-Software (2012). Top $10 \mathrm{Web}$ Conferencing Software Vendors Revealed. Recuperado de http://landing.businesssoftware.com/top-10-web-conferencingve ndors.ph p ? track $=1681 \&$ utm source $=$ webconferencing.org\&utm_med ium=banner\&atm_term $=\& u$ utm_term

Cacheiro González, M.L. (2011). Recursos educativos TIC de información, colaboración y aprendizaje. Revista Píxel-Bit. Revista de Medios y Educación, 39, 69-81. Recuperado de http://acdc.sav.us.es/pixelbit/images/ stories/p39/06.pdf.

Castillo Arredondo, S. \& Cabrerizo Diago, J. (2005): Formación del profesorado en Educación Superior, Didáctica y Currículum, volumen I. Madrid: McGraw Hill.

Cisco Webex (2012). Administración del sitio: Guía del usuario. Recuperado de https:/ /ciad.webex.com/docs/T27LD/common_docs/ es_ES/siteadmin/man/wx_siteadmin_ug.pdf.

Consejo Nacional de Ciencia y Tecnología -CONACYT- (Agosto, 2012). Programa nacional de posgrados de calidad marco de referencia para la evaluación y seguimiento de programas de posgrado en las modalidades a distancia y mixta consejo nacional de ciencia y tecnología subsecretaría de educación superior. Recuperado de http://www.conacyt.gob.mx/ FormacionCapitalHumano/Documents/ PNPC/Marco_Referencia_ NoEscolarizada.pdf

Gallegos Arrufat, M.J., Gámiz Sánchez, V. \& Gutiérrez Santiuste, E. (2010). El futuro docente ante las competencias en el uso de las tecnologías de la información y comunicación para enseñar. Edutec. Revista electrónica de tecnología educativa, 34, 118. Recuperado de http://edutec.rediris.es/ Revelec2/Revelec34/pdf/Edutece_n34_Gallego_Gamiz_Gutierrez.pdf.

González Isasi, R.M. (2010). Estrategias de formación de profesores para el uso didáctico de la tecnología. Revista Píxel-Bit. Revista de Medios y Educación, 37, 197-208. Recuperado de http://www.sav.us.es/pixelbit/ pixelbit/articulos/n37/16.html.

Graham, D. (1993). Quick Solutions to Great Layouts. Cincinnati. Ohio: North Ligth.

McAnally-Salas, L. (2005). Diseño educativo basado en las dimensiones del aprendizaje. Apertura, 5(1), 31-43.

McIsaac, M.S. \& Gunawardena C.N. (1996). Distance education. En D.H. Jonassen (ed.) Handbook of research for educational communications and technology. (pp. 403437). Nueva York: Simon and SchusterMacmillan.

Marcos, M. del S. (2010). Procesos y actividades generales de la universidad virtual del tecnológico de Monterrey. Revista digital la educación, 142. Recuperado de http://www.educoea.org/portal/ La_Educacion_Digital/laeducacion_142/ estudios/Procesosyactividades generalesdelaUniversidadVirtua $1 \mathrm{de} 1 \mathrm{Tec}$ o $1 \% \mathrm{C} 3 \% \mathrm{~B} 3 \mathrm{~g}$ i code M onterrey_MarcosMarcos_1.pdf. 
Salmon, G. (2004). E-actividades. El factor clave para una formación en línea activa. Barcelona: UOC.

Sánchez Rojo, I.J (2009). Plataforma Educativa Moodle: Administración y Gestión. México: Alfaomega/Ra-Ma.

Schwier, R.A. \& Misanchuk, E.R. (1993). Interactive Multimedia Instruction. Englewood Cliffs, N.J.: Educational Technology Publications.

Universidad Nacional de Educación a Distancia. UNED (2010). Cómo diseñar y ofertar cursos en línea. Consideraciones generales. Universidad Estatal a Distancia. España: PAL-PACE.

Urbina, S., Arrabal, M., Martín, A., Ordinas, C., Pons, S. \& Rodríguez, S. (2010). Análisis de herramientas de videoconferencia de escritorio para la docencia en pequeño grupo y la tutoría en entornos de educación superior. XIII Congreso Internacional Edutec 2010: E-Learning 2.0: Enseñar y Aprender en la Sociedad del Conocimiento. Bilbao, España.

WebConferencing (2011). Web Conferencing Buyer's Guide - Par t 1 Must have Features of Web Conferencing Software. Recuperado de http:// webconferencing.org/offer/ webconferencing-buyers-guide-part-1-musthave-features-of-web-conferencingsoftware/.

Fecha de recepción: 14-08-2013

Fecha de evaluación: 18-09-2013

Fecha de aceptación: 05-11-2013 